\title{
Development and characterization of the microsatellite markers for the Neptune whelk, Neptunea cumingii
}

Peizhen Ma ( $\nabla$ mapeizhen@qdio.ac.cn )

Institute of Oceanology Chinese Academy of Sciences https://orcid.org/0000-0002-4471-8191

Tao Zhang

Institute of Oceanology Chinese Academy of Sciences

Haizhou Li

Shandong Fuhan Marine Technology Co., Ltd

\section{Haiyan Wang}

Institute of Oceanology Chinese Academy of Sciences https://orcid.org/0000-0002-6297-3591

\section{Short Report}

Keywords: microsatellite markers, Neptunea cumingii, genetic diversity, Illumina, high-throughput sequencing

Posted Date: March 3rd, 2022

DOI: https://doi.org/10.21203/rs.3.rs-1406657/v1

License: (c) (i) This work is licensed under a Creative Commons Attribution 4.0 International License.

Read Full License 


\section{Abstract}

\section{Background}

The Neptune whelk, Neptunea cumingii, is an economic species endemic to the East Asia Warm Temperate Biotic Subregion. The development of microsatellite markers will be beneficial to the assessment of genetic diversity and conservation of resources on this decreasing gastropod.

\section{Methods and Results}

The microsatellite markers were developed and characterized through Illumina high-throughput sequencing and capillary electrophoresis techniques. 11 polymorphic microsatellite loci were screened and validated. The observed heterozygosity and expected heterozygosity of each locus ranged from 0.0600 to 0.6508 and from 0.7380 to 0.9375 , respectively. The average Shannon's information index, polymorphism information content, F-Statistics and gene flow were 2.0828, 0.8325, 0.0519 and 4.5660, respectively. The genetic diversity analysis indicated a closer genetic distance between populations of Dalian and Lvshun.

\section{Conclusions}

The distribution pattern of polymorphic microsatellite loci reveals that the Yellow Sea Warm Current generates the high level of genetic connectivity in N. cumingii. The markers developed in this study are expected as informative for further analysis of genetic diversity in $\mathrm{N}$. cumingii.

\section{Introduction}

The Neptune whelk, Neptunea cumingii, is a large gastropod distributed regionally within the areas of the Yellow Sea warm current and the Tsushima warm current $[1,2,3]$. As an economically important species, the muscle of $N$. cumingii is rich in essential amino acids and unsaturated fatty acids and valued by consumers [4], despite food poisoning case of tetramine has been reported [2]. With adult whelk mainly in gravel and sand bottom, the bottom trawlers have been widely used to catch $N$. cumingii, resulting in a sharp decrease and threatened status of wild resources [3]. Consequently, to relieve stress of overfishing and protect the wild resources, research on $N$. cumingii has been carried out, mainly focused on the feeding habits [5], the reproductive biology $[1,6,7]$ and artificial breeding [8].

Genetic diversity is the scientific basis for germplasm resource protection [9]. Sui (2008) selected simple sequence repeats from close-relative gastropods and verified them in $N$. cumingii [10]. Later, short gene fragments, COXI and $C Y T B$, were used to probe into genetic diversity of six geographic groups of $N$. cumingii in China, with no significant differences among populations detected [11]. Besides, polymorphic microsatellite loci were isolated from a congeneric whelk, $N$. arthritica [12]. Here in this study, we developed and characterized the microsatellite markers for N. cumingii using high-throughput sequencing and capillary electrophoresis techniques, aiming to assist in population genetic studies on this increasingly scarce gastropod. 


\section{Materials And Methods \\ Development of microsatellite markers}

To construct the genomic library, nine individuals of $N$. cumingii from seas of Dalian (DL), Lvshun (LS) and Weihai (WH) were selected, with three individuals from each sampling site (Fig. 1) [13]. Genomic DNA was extracted from the cryopreserved foot muscles using the Tiangen DNA kit (DP324, Tiangen Biotech (Beijing) Co., Ltd., Beijing) following the manufacturer's instructions. The genomic DNA was then fragmented into $\sim 400$ bp to develop the genomic library. Eight probes, including $p(A G)_{10}, p(A C)_{10}$, $\mathrm{p}(\mathrm{AAC})_{8}, \mathrm{p}(\mathrm{AGG})_{8}, \mathrm{p}(\mathrm{ACG})_{8}, \mathrm{p}(\mathrm{AAG})_{8}, \mathrm{p}(\mathrm{ATCT})_{6}$, as well as $\mathrm{p}(\mathrm{ACAT})_{6}$, were adopted in capturing microsatellites from the library by the magnetic beads enriched method. The enriched genomic library was sequenced on the Illumina NovaSeq platform (Illumina, San Diego, CA, USA) at Shanghai Personalbio Biotechnology Co., Ltd using $2 \times 150$ bp paired-end sequencing mode. Raw reads were filtered by removing reads of which the length are $\leq 50 \mathrm{bp}$, or of low quality, or with 3' adaptors using AdapterRomoval 2.1.7 [14], and combined by FLASH 1.2.11 [15]. Microsatellites were calculated by motif types. The loci were then searched from all combined sequences by an online Microsatellite identification tool at http://pgrc.ipk-gatersleben.de/misa/. After deleting the microsatellites with flanking sequences $<20 \mathrm{bp}$, CDHIT software was used to cluster the remaining sequences. The SSR length polymorphism (SSLP) of each cluster was calculated by Perl program. Microsatellite markers for $N$. cumingii were developed from clusters with SSLP $\geqq 2$ and designed using Primer3 v2.3.6 [16]. The primer pairs were then screened by the following criterions: a) no motif types were the mononucleotide repeat motifs or compound repeat motifs, b) only one SSR in each read, c) the primer pairs should be within one cluster, d) each designed primer had to be supported by two primers in the clusters, and e) exact same primers were removed.

\section{Screening And Characterization Of Microsatellite Markers}

To check the amplified fragment length and specific amplification, 50 primer pairs were selected randomly. The PCR mixture contained $0.5 \mu \mathrm{L}$ each primer, $1.0 \mu \mathrm{L}$ template DNA, $10 \mu \mathrm{L} 2 \times$ Taq PCR MasterMix (PC1120, Beijing Solarbio Science \& Technology Co., Ltd., Beijing, China), and $8 \mu \mathrm{L} \mathrm{H}_{2} \mathrm{O}$ in a $20 \mu \mathrm{L}$ reaction system. Fragments were amplified using the following protocol: initial denature at $95^{\circ} \mathrm{C}$ for $5 \mathrm{~min}, 35$ cycles of $95^{\circ} \mathrm{C}$ for $30 \mathrm{sec}, 50^{\circ} \mathrm{C}$ for $30 \mathrm{sec}$, and $72^{\circ} \mathrm{C}$ for $30 \mathrm{sec}$, with a final extension at $72^{\circ} \mathrm{C}$ for $7 \mathrm{~min}$. The agarose gel electrophoresis was used here to detect the PCR products. Primer pairs with single, bright bands agreeing with expected fragment sizes were selected to be further tested.

\section{Genetic Diversity Analysis Of Microsatellite Markers}

11 pairs of primers with high quality were selected (Table 1 ) and applied in three populations from Dalian (40 individuals), Lvshun (20 individuals) and Weihai (27 individuals). Genomic DNA was extracted as describe above and amplified with fluorescent labeled polymorphic microsatellite markers. The PCR mixture contained 1.0 $\mu \mathrm{L}$ each primer, $1.0 \mu \mathrm{L}$ template DNA, $2.0 \mu \mathrm{L}$ 10*buffer, $0.5 \mu \mathrm{L}$ dNTP, $0.5 \mu \mathrm{L}$ Taq DNA 
Polymerase and $14 \mu \mathrm{L} \mathrm{H}_{2} \mathrm{O}$ in a $20 \mu \mathrm{L}$ reaction system. Fragments were amplified using the protocol described above with corresponding annealing temperature. The products were purified and capillary electrophoresis [17] was conducted on ABI 3730XL DNA Analyzer (Applied Biosystems, Foster City, CA, USA).

Table 1

Characteristics of eleven microsatellite loci from N. cumingii developed in this study

\begin{tabular}{|c|c|c|c|c|c|}
\hline Locus & Primer sequence $\left(5^{\prime}-3^{\prime}\right)$ & $\begin{array}{l}\text { Repeat } \\
\text { motif }\end{array}$ & $\begin{array}{l}\text { Size range } \\
\text { (bp) }\end{array}$ & $\begin{array}{l}\mathrm{Ta} \\
\left({ }^{\circ} \mathrm{C}\right)\end{array}$ & $\begin{array}{l}\text { Fluorescence } \\
\text { labeling }\end{array}$ \\
\hline \multirow[t]{2}{*}{ NC01 } & F: AAAACCCTTTGATGTTTCCAG & $(A A G) n$ & $141-162$ & 59 & HEX \\
\hline & R: TGCTGTCCAGCTTGGTTATG & & & & \\
\hline \multirow[t]{2}{*}{ NC02 } & F: CCTCGACCCAGATGCTATTG & (TCT)n & $269-299$ & 60 & FAM \\
\hline & R: TTTTTCGGAAGAGTAGGCCA & & & & \\
\hline \multirow[t]{2}{*}{$\mathrm{NCO3}$} & F: TGGAGGAACGGGTGTCTAAT & $($ TGTA)n & $202-234$ & 60 & FAM \\
\hline & R: TTTACATCCCTCACCCGCTA & & & & \\
\hline \multirow[t]{2}{*}{$\mathrm{NCO} 4$} & F: СССААТТTТСТTТСССТТТСТT & $(A G) n$ & $163-189$ & 59 & HEX \\
\hline & R: GCCTTTTTCGGAATAAACCA & & & & \\
\hline \multirow[t]{2}{*}{ NC05 } & F: TGGCAACTTGGATCAGTTCTC & $(A G) n$ & $200-224$ & 60 & FAM \\
\hline & R: CACTTGATTGAGTCCTCGCA & & & & \\
\hline \multirow[t]{2}{*}{ NC06 } & F: TCCACTGGCCCTGTATCTCT & $(\mathrm{AGAT}) \mathrm{n}$ & $260-300$ & 60 & HEX \\
\hline & $\begin{array}{l}\text { R: } \\
\text { CATCCATCTGTCTATCTCTCTATCCA }\end{array}$ & & & & \\
\hline \multirow[t]{2}{*}{ NC07 } & F: GTAGCTGTTGACCCAAACCC & $(A A G) n$ & $272-302$ & 60 & TAMRA \\
\hline & R: GAATGGACCAAAGCCAAAGA & & & & \\
\hline \multirow[t]{2}{*}{ NC08 } & F: СCTTCTTCGTCTTGTTCTTGTTC & $($ TTC)n & $356-385$ & 59 & FAM \\
\hline & R: TTCATTTTGTTTCGTCAACCA & & & & \\
\hline \multirow[t]{2}{*}{ NC09 } & F: ATCCGCTCCCAAAGTTAGGT & $(A A G) n$ & $192-143$ & 59 & HEX \\
\hline & R: ACTGTTTCCCCAGCATGAGT & & & & \\
\hline \multirow[t]{2}{*}{ NC10 } & F: CCCTTGTTCTTCACCAAAAATC & $(A G) n$ & $218-230$ & 60 & TAMRA \\
\hline & R: TGGTGCATAATGTGCTTTCTC & & & & \\
\hline \multirow[t]{2}{*}{ NC11 } & F: TGAAGATATCTCCCACACTTGG & $(A A G) n$ & $246-261$ & 60 & HEX \\
\hline & R: CTTGTTCCGCTTCCACTGTT & & & & \\
\hline
\end{tabular}


Results were analyzed by GeneMarker V2.2.0 to read the size and signal value of the fragments. The observed number of alleles $(\mathrm{Na})$, effective number of alleles $(\mathrm{Ne})$, observed heterozygosity $(\mathrm{Ho})$, expected heterozygosity $(\mathrm{He})$, Shannon's information index (I) and F-Statistics (Fst) were evaluated by PopGene software verson 1.31, as well as Nei's Unbiased measures of genetic identity and genetic distance among three populations [18]. The gene flow $(\mathrm{Nm})$ was estimated following $N m=0.25(1-F s t) / F s t$. And polymorphism information content (PIC) was calculated using the formula proposed by Bostein et al [19].

\section{Results And Discussion}

By Illumina high-throughput sequencing of nine individuals of $N$. cumingii, 8,776,062 raw reads were obtained with 1,316 million bp total bases. The average GC content was $41.86 \%$. Totally, 7,339,720 highquality reads were obtained, accounting for $83.63 \%$ raw reads. $2,497,115$ combined pairs were assembled, from which a total of $30,31,455$ microsatellites were identified. 851,479 combined pairs had more than one microsatellite. Most microsatellites were mononucleotide repeat motifs, accounting for $94.55 \%$ all microsatellites. 7875 microsatellites (4.50\%) were dinucleotide repeat motifs, and $1135(0.65 \%)$ and 347 $(0.20 \%)$ were trinucleotide and tetranucleotide repeat motifs, respectively. The cluster analysis revealed 242,751 clusters in total, of which 51,467 clusters $(21.20 \%$ ) showed length polymorphism ( $\geqq 2$ ) across 9 individuals. Thereinto, 27,626 clusters $(11.38 \%)$ had two lengths. After the primer pairs were filtered, 1297 primer pairs with SSLP from 2 to 4 were obtained.

A total of 11 primer pairs were validated in 87 individuals from three populations (Table 2). The $\mathrm{Na}$ and $\mathrm{Ne}$ ranged from 6 to 13 and from 3.7478 to 14.4273, respectively. Both Ho and He were high, with means of 0.4355 and 0.8447 for each locus, respectively. The Shannon's information index values varied from 1.5955 to 2.4490 . The $P I C$ values of each locus varied from 0.7296 to 0.9301 , with all greater than 0.5 , suggesting that all the eleven microsatellite loci were highly polymorphic and valuable for genetic diversity studies [19]. The average $F s t$ and $N m$ values were 0.0519 and 4.5660, indicating little genetic differentiation and abundant gene flow [20]. In addition, the maximum value (0.8332) and minimum value (0.6096) of Nei's genetic identity occurred between populations of Lvshun and Dalian and between Lvshun and Weihai, respectively, corresponding to the minimum and maximum genetic distance values of 0.1825 and 0.4949. The distribution pattern reveals that the Yellow Sea Warm Current is the drive that leads to the high level of genetic connectivity in N. cumingii. In conclusion, the 11 microsatellite markers developed and characterized in this study provide high level of genetics, and these markers will be useful for further analysis of genetic diversity in $N$. cumingii. 
Table 2

Genetic diversity based on 11 microsatellite loci of N. cumingii

\begin{tabular}{|c|c|c|c|c|c|c|c|c|c|}
\hline Locus & $\begin{array}{l}\text { Sample } \\
\text { size }\end{array}$ & $\mathrm{Na}$ & $\mathrm{Ne}$ & Ho & $H e$ & I & $P I C$ & Fst & $N m$ \\
\hline NC01 & 152 & 8 & 5.4516 & 0.5263 & 0.8220 & 1.8977 & 0.8113 & 0.0575 & 4.0993 \\
\hline $\mathrm{NCO} 2$ & 154 & 9 & 3.7478 & 0.4545 & 0.7380 & 1.6868 & 0.7311 & 0.0522 & 4.5383 \\
\hline NCO3 & 148 & 9 & 3.7909 & 0.3378 & 0.7412 & 1.7077 & 0.7296 & 0.0648 & 3.6100 \\
\hline $\mathrm{NCO} 4$ & 126 & 13 & 10.2558 & 0.6508 & 0.9097 & 2.4490 & 0.9002 & 0.0449 & 5.3179 \\
\hline NC05 & 164 & 12 & 9.8089 & 0.5854 & 0.9036 & 2.3744 & 0.8968 & 0.0296 & 8.2066 \\
\hline NC06 & 114 & 11 & 7.3757 & 0.5088 & 0.8721 & 2.1666 & 0.8602 & 0.0302 & 8.0269 \\
\hline NC07 & 136 & 11 & 8.1337 & 0.3971 & 0.8836 & 2.2082 & 0.8733 & 0.0337 & 7.1696 \\
\hline NC08 & 132 & 11 & 9.2190 & 0.6364 & 0.8983 & 2.3018 & 0.8902 & 0.0404 & 5.9400 \\
\hline NCO9 & 138 & 17 & 14.4273 & 0.3333 & 0.9375 & 2.7434 & 0.9301 & 0.0256 & 9.5205 \\
\hline NC10 & 100 & 7 & 5.2083 & 0.3000 & 0.8162 & 1.7794 & 0.7945 & 0.0657 & 3.5546 \\
\hline NC11 & 100 & 6 & 4.2017 & 0.0600 & 0.7697 & 1.5955 & 0.7403 & 0.1445 & 1.4805 \\
\hline Mean & 133 & 10.3636 & 7.4201 & 0.4355 & 0.8447 & 2.0828 & 0.8325 & 0.0519 & 4.5660 \\
\hline \multicolumn{10}{|c|}{$\begin{array}{l}\text { Na: observed number of alleles, Ne. effective number of alleles, Ho: observed heterozygosity, He. } \\
\text { expected heterozygosity, I: Shannon's information index, }\end{array}$} \\
\hline
\end{tabular}

\section{Declarations}

\section{Acknowledgements}

We thank Cong Zhou, Xiaolong Wang and Zhi Hu for assistance in collecting the experimental materials. We appreciate the help in data processing from Yue Tan of the Ocean University of China.

\section{Funding}

This work was supported by the National Key R\&D Program of China (No. 2019YFD0901303, 2019YFD0900800), the China Agriculture Research System of MOF and MARA, the Major Scientific and Technological Innovation Project of Shandong Provincial Key Research and Development Program (No. 2019JZZY020708), the Industry Leading Talents Project of Taishan Scholars (Recipient: Tao Zhang), the 'Double Hundred' Blue Industry Leader Team of Yantai (Recipient: Tao Zhang) and the Science and Technology Service Network Initiative, Chinese Academy of Sciences (No. KFJ-STS-QYZD-189).

\section{Competing Interests}


The authors have no relevant financial or non-financial interests to disclose.

\section{Author contributions}

HW and TZ designed the experiment. Material preparation was performed by HL. Data collection and analysis were performed and the first draft of the manuscript was written by PM. All authors commented on previous versions of the manuscript and approved the final manuscript.

\section{Ethics approval}

This study did not require ethics approval.

\section{Consent to participate}

Informed consent was obtained from all individual participants included in the study.

\section{Consent to publish}

The authors consent to publish all the data in this study.

\section{References}

1. An JE, Choi JD, Ryu DK (2015) Age and growth of the Neptunea (Barbitonia) arthritica cumingii in the West Sea of Korea. The Korean Journal of Malacology 31: 165-170.

http://dx.doi.org/10.9710/kjm.2014.30.1.25

2. Nishikawa T, Doi K, Tsujimura K, Hamano T (2009) Food poisoning case of tetramine from eating Neptunea arthritica cumingii. Annual Report of Nagasaki Prefectural Institute of Public Health and Environmental Sciences 55: 118-120.

3. Guo D, Liu X, Wang A et al (2015) Stock distribution of whelk Neptunea arthritica Crosse in Liaodong Bay. Fisheries Science 34: 718-721. http://doi.org/10.16378/j.cnki.1003-1111.2015.11.009

4. Hao Z, Wang Y, Yu Y et al (2016) Analysis and evaluation of nutritive composition in the muscle of Neptunea arthritica cumingii Crosse (Gastropoda: Buccinidae). Journal of Dalian Ocean University 37: 66-70.

5. Yang M, Feng J, Yu Z et al (2020) Comparative analysis of the feeding habits of Rapana venosa and Neptunea arthritica cumingii near Zhangzi Island, China, based on stable isotope ratios and fatty acid profiles. Aquac Res 52: 1846-1854. http://doi.org/10.1111/are.15033

6. Hao Z, Liu H, Yu Y et al (2021) Reproductive characteristics and variations in the biochemical composition of Neptunea arthritica cumingii Crosse through embryonic development. Aquac Res 52: 1-11. http://doi.org/10.1111/are.14790 
7. Lombardo RC, Goshima S (2010) Female copulatory status and male male choice in Neptunea arthritica (Gastropoda: Buccinidae). J Mollus Stud 76: 317-322. http://doi.org/10.1093/mollus/eyq015

8. Yu Y, Li J, Wu W, Hao Z (2019) Research development of reproductive biology and artificial breeding technology of Neptunea cumingii. Hebei Fisheries 4: 54-56. http://doi.org/10.3969/j.issn.10046755.2019.04.014

9. Xia Y, Guo P, Li R et al (2014) Analysis of genetic diversity and population structure using SSR markers in tobacco. Advanced Materials Research 850-851: 1243-1246.

https://doi.org/10.4028/www.scientific.net/AMR.850-851.1243

10. Sui N, Hou L, Dong C et al (2008) Optimization of fingerprint on simple sequence repeats (SSR) of conch Neptunea cumingi. Fisheries Science 27: 199-202. http://doi.org/10.16378/j.cnki.1003-

1111.2008.04.006

11. Zhang D, Wang Y, Li Z et al (2021) Genetic diversity of mitochondial COXI and CYTB genes in Neptune whelk Neptunea cumingii. Journal of Dalian Ocean University 36: 612-619. http://doi.org/10.16535/j.cnki.dlhyxb.2020-285

12. Azuma N, Miranda RM, Goshima S, ABE S (2009) Polymorphic microsatelliate markers isolated from the neptune whelk Neptunea arthritica. Mol Ecol Resour 9: 120-121. http://doi.org/10.1111/j.17550998.2008.02179.x

13. Schlitzer R (2021) Ocean Data View http://odv.awi.de

14. Lindgreen S (2012) AdapterRemoval: easy cleaning of next-generation sequencing reads. BMC Res Notes 5: 337. http://doi.org/10.1186/1756-0500-5-337

15. Magoč T, Salzberg SL (2011) FLASH: fast length adjustment of short reads to improve genome assemblies. Bioinformatics 27: 2957-2963. http://doi.org/10.1093/bioinformatics/btr507

16. Untergasser A, Cutcutache I, Koressaar T et al (2012) Primer3-new capabilities and interfaces. Nucleic Acids Res 40: e115. http://doi.org/10.1093/nar/gks596

17. Vemireddy LR, Archak S, Nagaraju J (2007) Capillary electrophoresis is essential for microsatellite marker based detection and quantification of adulteration of basmati rice (Oryza sativa). J Agric Food Chem 55: 8112-8117. http://doi.org/10.1021/jf0714517

18. Nei M (1978) Estimation of average heterozygosity and genetic distance from a small number of individuals. Genetics 89: 583-590. http://doi.org/10.1007/BF00155576

19. Botstein D, White RL, Skolnick M, Davis RW (1980) Construction of a genetic linkage map in man using restriction fragment length polymorphisms. Am J Hum Genet 32: 314-331. 
20. Reichow D, Smith MJ (2001) Microsatellites reveals high levels of gene flow among populations of the California squid Loligo opalescens. Mol Ecol 10: 1101-1109. http://doi.org/10.1046/j.1365294x.2001.01257.x

\section{Figures}



Figure 1

A map of sampling sites of the Neptune whelk, N. cumingii, in this study. DL: Dalian, LS: Lvshun, WH: Weihai, YSWC: Yellow Sea Warm Current, BCC: Bohai Coastal Current, YSCC: Yellow Sea Coastal Current. 